\title{
Clinical features and prognostic factors of patients with COVID-19 in Henan Province, China
}

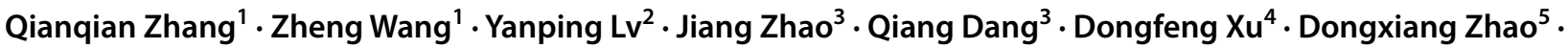 \\ Haiyang Liu ${ }^{1} \cdot$ Ziqi Wang $^{1} \cdot$ Xingru Zhao $^{1} \cdot$ Zhiwei Xu $^{6} \cdot$ Xiaoju Zhang $^{1}$
}

Received: 22 December 2020 / Accepted: 28 January 2021 / Published online: 13 February 2021

(c) Japan Human Cell Society 2021

\begin{abstract}
Since December 2019, the novel coronavirus SARS-CoV-2 pandemic (COVID-19) outbroke in Wuhan and spread in China. Here we aimed to investigate the clinical and radiological characteristics of COVID-19 cases. We collected and analyzed the clinical data of 172 hospitalized cases of COVID-19 who were diagnosed via qRT-PCR of nasopharyngeal swabs during January 2020 and February 2020. The chest images were reviewed by radiologists and respirologists. The older patients with COVID-19 in Henan Province had more severe disease and worse prognosis. The male sex, smoking history and Wuhan exposure of patients are not related to the severity or prognosis of COVID-19. Family gatherings were showed among 26.7\% of patients. A greater proportion of patients in the severe group suffer from combined chronic diseases. CT results showed that most patients had bilateral lung lesions and multiple lung lobes. The lungs of severe patients are more damaged. Both the infection range and inflammatory factor levels are related to the poor prognosis. Antiviral drugs, immunoglobulin and traditional Chinese medicine are mainly used for the treatment of COVID-19 patients. The discharge rate of COVID-19 patients was $93.0 \%$, and the mortality rate was $2.3 \%$. Case type, lymphocyte ratio grade, and respiratory failure at admission are risk factors for poor prognosis, except for the number of infiltrating lung lobes. The results showed that severe disease process, lymphopenia and respiratory failure are risk factors for the COVID-19.
\end{abstract}

Keywords COVID-19 $\cdot$ SARS-CoV-2 $\cdot$ Ground glass opacity $\cdot$ Lymphopenia $\cdot$ Mortality

Qianqian Zhang, Zheng Wang have equally contributed.

Xiaoju Zhang

15837101166@163.com

1 Department of Respiratory and Critical Care Medicine, Zhengzhou University People's Hospital, Henan Provincial People's Hospital, No. 7 Weiwu Road, Zhengzhou 450003, Henan, China

2 Department of Respiratory and Critical Care Medicine, Zhoukou Central Hospital, Zhoukou 466000, Henan, China

3 Department of Respiratory and Critical Care Medicine, Nanyang Central Hospital, Nanyang 473000, China

4 Department of Respiratory and Critical Care Medicine, The First Affiliated Hospital of Nanyang Medical College, Nanyang 473000, China

5 Department of Respiratory and Critical Care Medicine, Dengzhou Central Hospital, Dengzhou 474100, China

6 Clinical Research Service Center, Henan Provincial People's Hospital, Zhengzhou University People's Hospital, Zhengzhou 450003, Henan, China

\section{Introduction}

Since December 2019, a novel respiratory virus pandemic outbroke in Wuhan, Hubei Province, China [1-8]. It has spread to other provinces in China as well as to other countries, which infected more than 400,000 people and was responsible for over 18,000 deaths worldwide till Mar 24th, 2020 , as the victim numbers tend to be rising despite measures undertaken for controlling it. One of the utmost of stopping the pandemic is to fully recognize, isolate and cure the patients together with a more comprehensive understandings of both the disease and the virus.

The virus was identified as the seventh member of enveloped RNA coronavirus (subgenus sarbecovirus, Orthocoronavirinae subfamily) by high-throughput sequencing, and was named as SARS-CoV-2 $[9,10]$. Pneumonia associated with SARS-CoV-2 infection is named COVID-19 [10], which resembles some clinical features of severe acute respiratory syndrome (SARS) and middle east respiratory syndrome (MERS) [1-8]. Presumably, the 
fatality rate of COVID-19 is approximately $1.5 \%$ [1-8], which is less fatal than SARS (11\%) and MERS (35\%), while in some countries the fatality rate may rise to $6-8 \%$. The contagion of SARS-CoV-2 seems to be much more difficult to be dealt with, as revealed by 10-20 times higher affinity of spike glycoprotein to ACE2 compared to SARS [11], an estimated R0 of 3.0-4.0 [6] and by reported cases of insidious contagion by asymptomatic carriers as well as familial, in-prison and in-hospital transmission $[6,12,13]$. Recent studies have shed light on the characteristics and prognosis of patients with COVID-19. The preliminary report by Huang et al. [1] highlighted the first 41 Wuhan patients with their clinical features and courses, laboratory test results including the existence of detectable serum viral RNA and elevated serum inflammatory cytokine levels, high risks of ARDS and ICU admission, and unfavorable outcomes in critically ill patients. Yang et al. [14] demonstrated that older age and the development of ARDS predict poor outcomes in critically ill patients with COVID-19. Guan et al. [8] performed a large cohort analysis of 1099 COVID-19 patients that were treated in 31 provinces or provincial municipalities all over China. The death rates of all patients, non-severe patients and severe patients were $1.4 \%, 0.1 \%$ and $8.1 \%$, respectively, which were predicted by disease severity parameters including oxygen saturation, respiratory rate, blood leukocyte/lymphocyte count and chest X-ray/CT manifestations. A recent epidemiological analysis of 72314 cases (44 672 cases or $61.8 \%$ were diagnosed by nucleic acid test) revealed that severe or critically ill patients account for $19.1 \%$ of all patients with COVID-19; the gross fatality rate was $2.3 \%$, with $81 \%$ deaths occurred in 60 years old or more, and complications/comorbidities increase the morality [6].

There are 107 million residents in Henan Province approximately, thousands of whom reside, work or study in Wuhan and return back home to celebrate the Spring Festival during January 2020 to February 2020. For instance, more than 36,000 people return back from Wuhan to Nanyang, a city of Henan Province that borders Hubei Province in the northeast. COVID-19 patients and asymptomatic SARSCoV-2 carriers have been identified among the returning home personnel, which have caused local residents to be infected in Nanyang. The situation is similar to that of Zhejiang Province, as reported by $\mathrm{Xu}$ et al. [7] that none of the cases has never been to the Wuhan seafood market. Supposedly the epidemiological and clinical features might be different among the patients with different exposure histories. Here we sought to investigate the clinical, laboratory, radiological characteristics of the patients diagnosed and treated in Nanyang city, and to seek the differences between patients with different exposure histories and clinicoradiological characteristics, providing insights into the prevention and treatment of COVID-19.

\section{Methods}

\section{Data sources and processing}

This is a retrospective study in Henan Province, China. We sought the medical records of Henan Provinical People's Hospital, Zhoukou Central Hospital, Nanyang Central Hospital, the First Affiliated Hospital of Nanyang Medical College and Dengzhou Central Hospital for cases with COVID-19. These four hospitals include a provincial hospital, three municipal hospitals that covered 11 million people in Nanyang City and 8 million people in Zhoukou City, and a county hospital (Dengzhou Central Hospital) that serves 1400 thousand people. These hospitals represent the typical three grades of the healthcare system (provincial, municipal and county level) in Henan Province that were qualified to treat patients with COVID19. Patients got fully access to the medical facilities as needed, and all the Medicare fees were covered by the central and local government. Cases were suspected to have COVID-19 in those who had a recent exposure history to Wuhan or to confirmed COVID-19 patients in the previous 2 weeks, and was presented with fever and/or respiratory symptoms (e.g. cough and dyspnea), or having fever/respiratory symptoms and typical radiological abnormalities (such as multiple ground-glass opacity) on CT or chest $\mathrm{X}$-ray. The suspected cases were closely followed up and subjected to real-time reverse transcriptase-polymerase chain reaction (RT-PCR) assay for nasopharyngeal swab specimens. Patients were diagnosed based on the WHO interim guidance [15]. Suspected cases were excluded after two consecutive RT-PCR assays were negative (not collected on the same day) and a careful discussion by a multidisciplinary discussion involving respirologists, epidemiologists, radiologists and intensive care experts. Doctors of the confirmed cases were fully accessible to all available medical resources including intensive care, and some patients were transferred to regional medical centers or university-affiliated hospitals for ECMO care. We included laboratory-confirmed cases for further analysis. Written consent was waived by the IRB considering that this was a retrospective study. The research was approved by the academic committees of each hospital, Zhengzhou University and Nanyang Medical College.

The epidemiological data, the laboratory test result data, the radiological reports and radiological data were acquired from electronic medical record systems of each hospital. Photos were taken and read by undergraduates or medical doctor candidates and recorded by being entered into a database, then checked by respiratory clinicians. Exposure history, disease onset, time to the first negative nasopharyngeal swab nucleic acid test, time to afebrile and 
time to discharge were documented. The usage of antiviral drugs, anti-inflammatory drugs, respiratory supplementary treatments [oxygen supply through a nasal duct or facial mask, high-flow nasal cannula oxygen (HFNC), noninvasive ventilation (NIV), invasive mechanical ventilation (IMV), extracorporeal membrane oxygenation (ECMO)], continuous renal replacement therapy (CRRT), the length of ICU stay, as well as complications and comorbidities were also recorded. The radiological data (expressed as DICOM or JPEG format) were copied from the electronic imaging systems of each hospital and reviewed by a panel of radiologists and respirologists. Further radiological analysis was performed using CT image processing software.

The severity of COVID-19 was defined based on the international guidelines for community-acquired pneumonia [16]. Briefly, mild COVID-19 was defined by the presence of minimal symptoms and absence of radiological abnormalities; common COVID-19 was defined by the presence of fever and/or respiratory symptoms and radiological alterations of pneumonia; severe COVID-19 met one or more of these criteria: respiratory distress and respiratory rate $\geq 30 \mathrm{bpm}$, oxygen saturation $<93 \%$ at rest on room air, $\mathrm{PaO}_{2} / \mathrm{FiO}_{2} \leq 300 \mathrm{mmHg}$; critically ill patients met one or more of these criteria: respiratory failure that requires mechanical ventilation, presence of shock, complicated with other organic failure that requires intensive care in the ICU. The time to afebrile was defined as the duration from the days of disease onset to the first day when the patient was no longer febrile. The time to nucleic acid negative was defined as the duration from disease onset to those of twice rhinopharyngeal swab nucleic acid tests appearing negative, i.e. meet the criteria of isolation cessation. Further inquiry and communication were done between data processor and analyzer and attending physicians whenever there was missing data, or anything question that may interfere were data analyzing. Some patients who met the criteria of non-severe COVID-19 were also treated in the ICU or respiratory ICU, while some patients who met the criteria of severe COVID19 were treated in common respiratory wards with HFNC or NIV till being transferred to the ICU. So we used a composite endpoint of intensive care instead of ICU stay, which refers to the use of non-invasive ventilation (NIV), invasive mechanical ventilation (IMV), extracorporeal membrane oxygenation (ECMO) or death. All data were dated back to March 15th, 2020. For patients still on treatment, parameters such as length of hospital stay alike refer to hospitalization till March 15th, 2020.

\section{Laboratory test}

The hospitals utilized next-generation sequencing and realtime RT-PCR assay for the detection of COVID-19. Briefly, throat-swab specimens were obtained from patients with fever, cough or dyspnea every other day and subjected to PCR examination. Blood examinations were routinely performed, including complete blood count, coagulation profile, serum biochemical tests (alanine aminotransferase, aspartate transaminase, procalcitonin, total bilirubin, hyperbilirubinemia, lactose dehydrogenase, creatinine and urea nitrogen.

\section{Computed tomography (CT) image}

LightSpeed Plus (GE, Medical System, Milwaukee, WI) and UCT 780 (United Imaging, Shanghai, China) were employed for scanning chest CT images with a tube voltage of $120 \mathrm{kV}$ combined with automatic tube current modulation (100-400 mA). Images were reconstructed with a slice thickness of $1.25 \mathrm{~mm}$ and an interval of $1.25 \mathrm{~mm}$, respectively. All patients underwent initial CT 3 days after the onset of symptoms. The interval time from initial to followup examination was 5 days.

\section{Differential diagnosis and confirmation of the SARS-CoV-2 virus infection}

SARS-CoV-2 was confirmed through RT-PCR assay of viral nucleic acid in nasopharyngeal swabs and was conducted in accordance with the protocol established by the World Health Organization [17]. The tests and further confirmation were executed by the coordination of the clinical laboratories of each hospital, the Nanyang Center for Disease Prevention and Control (CDC), the Zhoukou city CDC and the Henan Provincial CDC. Co-infections were excluded either by nasopharyngeal antigen test on influenza $\mathrm{A}$ and influenza $\mathrm{B}$, or serological tests on influenza $\mathrm{A}$, influenza B, parainfluenza virus, adenovirus, rhinovirus, respiratory syncytial virus, Chlamydia pneumoniae, Mycoplasma pneumoniae, Legionella pneumophila and Coxiella burnetii. Culture of sputum, bronchoalveolar lavage fluid, airway aspirates, blood, urine or duct tips was performed as necessary. Interferon- $\gamma$ release assay (IGRA), 1-3- $\beta$-D glucan test ( $\mathrm{G}$ test) and $\beta$-galactomannan test (GM test) were performed to exclude mycobacterium or fungi infections. Connective tissue disease, lung cancer or other diseases were excluded by consultation of the expert panels of each hospital based on clinical, laboratory and radiological data.

\section{Statistical analysis}

Continuous variables were descriptively analyzed as either means and standard deviations or medians with interquartile ranges. Further comparisons were performed by independent sample student $t$ test for 2 groups, one-way ANOVA and Mann-Whitney test for 3 or more groups or Wilcoxon ranksum test as appropriate (e.g. if the variance was uneven). 
Categorical variables were expressed as numbers and percentages and compared using chi-square tests and Fisher's exact tests. Cox regression analysis and Kaplan-Meier survival tests were used for analyzing the incubation period, time to clinically stable, time to first nucleic acid negative or other survival indices. We set up logistic multivariate regression models to explore the prognostic factors of complex unfavorable outcomes. Data were input and analyzed using SPSS software (version 17.0, IBM Inc., Armonk, NY) and GraphPad Prism software (version 8.0, GraphPadSystat Software Inc., San Diego, CA). Missing values were exempted from statistical analysis and expressed in the results. Two-tailed $P$ values of less than 0.05 are considered statistically significant.

\section{Results}

\section{Demographic, epidemiological and clinical characteristics}

The patient enrollment is outlined in Figure S1 and Figure S2. We retrieved medical records from 172 patients that were admitted with SARS-CoV-2 infection. CT images of seven cases were normal on their first CT, two of whom showed some extent of abnormalities on their later follow-up CT and were thus enrolled. Three patients were excluded for lacking some clinical data. The clinical characteristics are outlined in Table $1.78 .5 \%$ of all patients had a clear exposure history, and the median incubation period was 3.0 (95\% CI: 2.6-5.8, range: $1.0-17.0$ ) days. $45.3 \%$ and $34.6 \%$ of all patients had a recent Wuhan exposure history (reside or travel in Wuhan). $26.7 \%$ of all patients had familial cluster, i.e. one relative or more to be diagnosed with SARS-CoV-2 infection, and the average whole number of familial member infection was $2.2 \pm 1.6$ (95\% CI: $1.0-3.5$, range: $0-4)$.

The mean age was $47.9 \pm 18.3$ (range: $16-98$ ) years, and $46.5 \%$ were females. All patients have at least one symptom, and the most common symptoms were fever (94.2\%) and cough (68.6\%), followed by expectoration (41.9\%), fatigue $(36.0 \%)$ and dyspnea (32.6\%). $40.7 \%$ of all patients had at least one comorbidity, among which hypertension (18.6), diabetes (17.4\%) and coronary artery disease (7.6\%) were most prevalent. $121(70.3 \%)$ and $51(29.7 \%)$ patients were classified as non-severe and severe cases, respectively. Severe group differed from non-severe group in age, duration of fever, fatigue, cyanosis, and the number and type of comorbidities. 31 patients $(18.0 \%)$ reached the outcome of NIV, IMV, ECMO or death, and are distinguished in age, duration of fever, fatigue, cyanosis and comorbidities. Clinical characteristics are equally distributed in patients with or without a history of Wuhan residence.

\section{Radiological manifestations}

According to Fig. 1 and Table 2, the lesions of $77.3 \%$ of all cases were bilaterally distributed, with $65.1 \%$ of pure GGO, $5.8 \%$ of pure consolidation and $25.6 \%$ of a mixed type of GGO and consolidation. 3.5\% of all patients were CTnegative on admission. Pleural effusions were documented in $2.9 \%$ of all cases, $1.7 \%$ unilateral and $1.2 \%$ bilateral. Severe cases and those received intensive care have more lobes involved.

\section{Laboratory test results}

As shown in Table 2, leukopenia, lymphopenia and thrombocytopenia were documented in $32.6 \%, 76.7 \%$ and $7.0 \%$ of all patients, respectively. The serum levels of CRP, procalcitonin, aminotransferases (ALT or AST), creatine kinase MB subtype (CKMB), BNP/pro-BNP/pro-NT-BNP and D-dimer were elevated in $66.3,27.9 \%, 18.6 \%, 19.2 \%, 14.5 \%$ and $45.3 \%$, respectively. Severe group differed from non-severe group in oxygenation, lactate level, WBC, NEU, LYM and \%LYM, PCT, CRP, LDH, CK, BUN and D-dimer (all $P<0.05)$. Statistical significances between patients receiving intensive care or not are also documented in radiological involvement, oxygenation, lactate, WBC, NEU, LYM, \%LYM, CRP, PCT, AST, Hyperbilirubinemia, hypokalemia, hyponatremia, BNP and D-dimer. Patients with or without Wuhan residence history differ in AST, hypoproteinemia, $\mathrm{Hb}$ and PLT.

\section{Treatment, complications, outcomes and costs}

According to Table 3, Lopinavir/ritonavir and aerosol IFN$\alpha 1 \mathrm{~b}$ were most universally used, which account for $93.0 \%$ and $96.5 \%$ of all cases, respectively. Darunavir and remdevisir were used in $9.3 \%$ and $1.2 \%$ of all cases. $5.8 \%$ of patients underwent an antiviral regime shift because that initial treatment failed. Intravenous methylprednisolone was administered in $57 \%$ of all patients with an average duration of $(3.5 \pm 4.3)$ days and maximal daily doses of $0-160 \mathrm{mg}$. Intravenous immunoglobulin (IVIG), intravenous antibiotics and antifungals were administered in $39.0 \%, 98.8 \%$ and $7.0 \%$ of all patients, respectively. Oxygen therapy, high-flow nasal cannula oxygen therapy, NIV, IMV, ECMO was used in $61.6 \%, 7.6 \%, 11.6 \%, 7.0 \%, 17.4 \%$ and $3.5 \%$ of all cases, respectively. $30.8 \%$ of patients had a co-infection, $13.4 \%$ were bacteria, $5.2 \%$ fungi, $5 / 8$ another respiratory virus (3 cases of influenza A, 5 cases of influenza B, 1 case of both influenza A and B, 1 case of adenovirus), 1 Mycobacterium tuberculosis. The mean length of stay, the time from disease onset to the first visit, the time from disease onset to confirmation, the time from confirmation to initial antiviral treatment, the time (from antiviral treatment) to afebrile, the time 


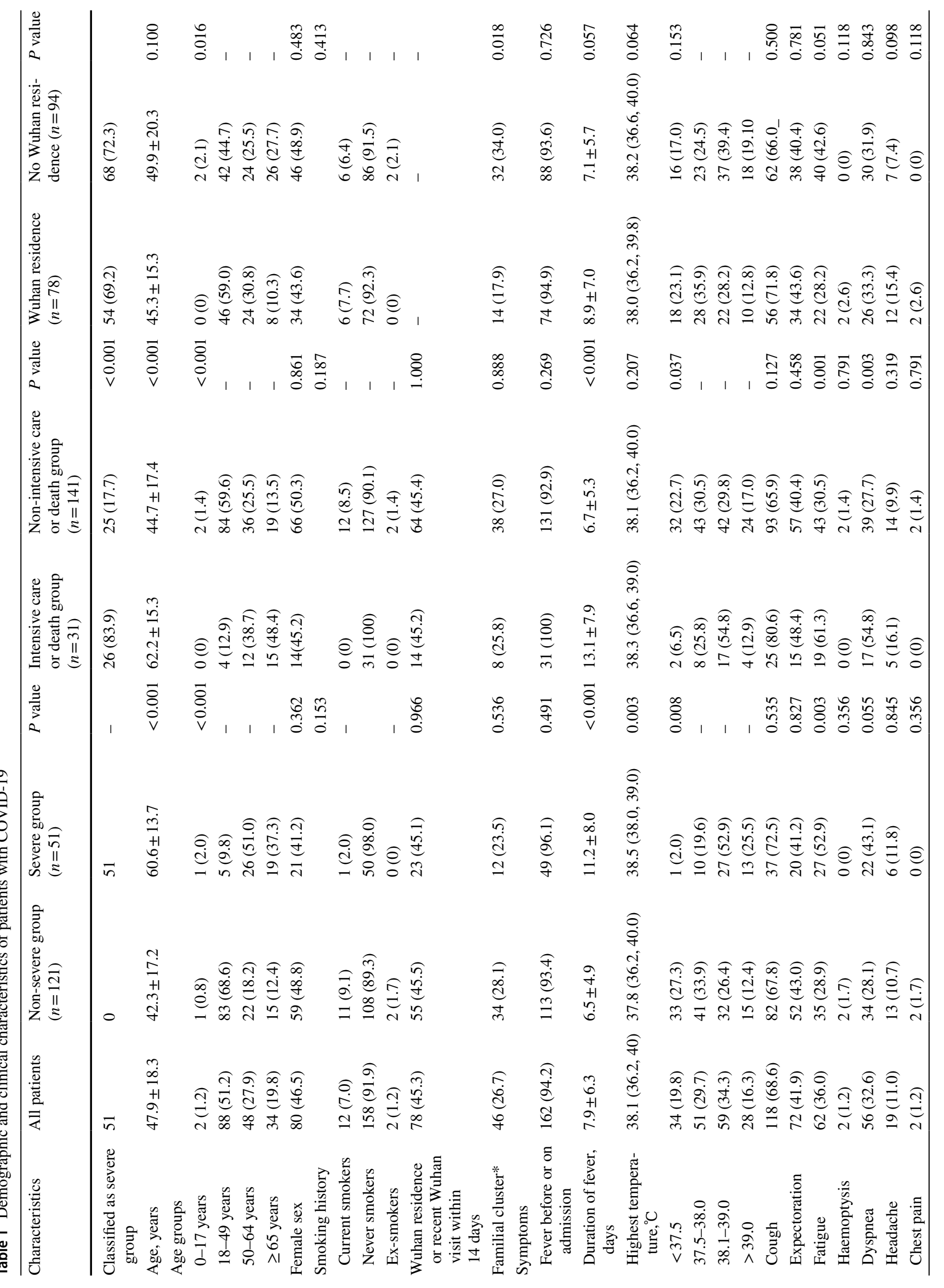




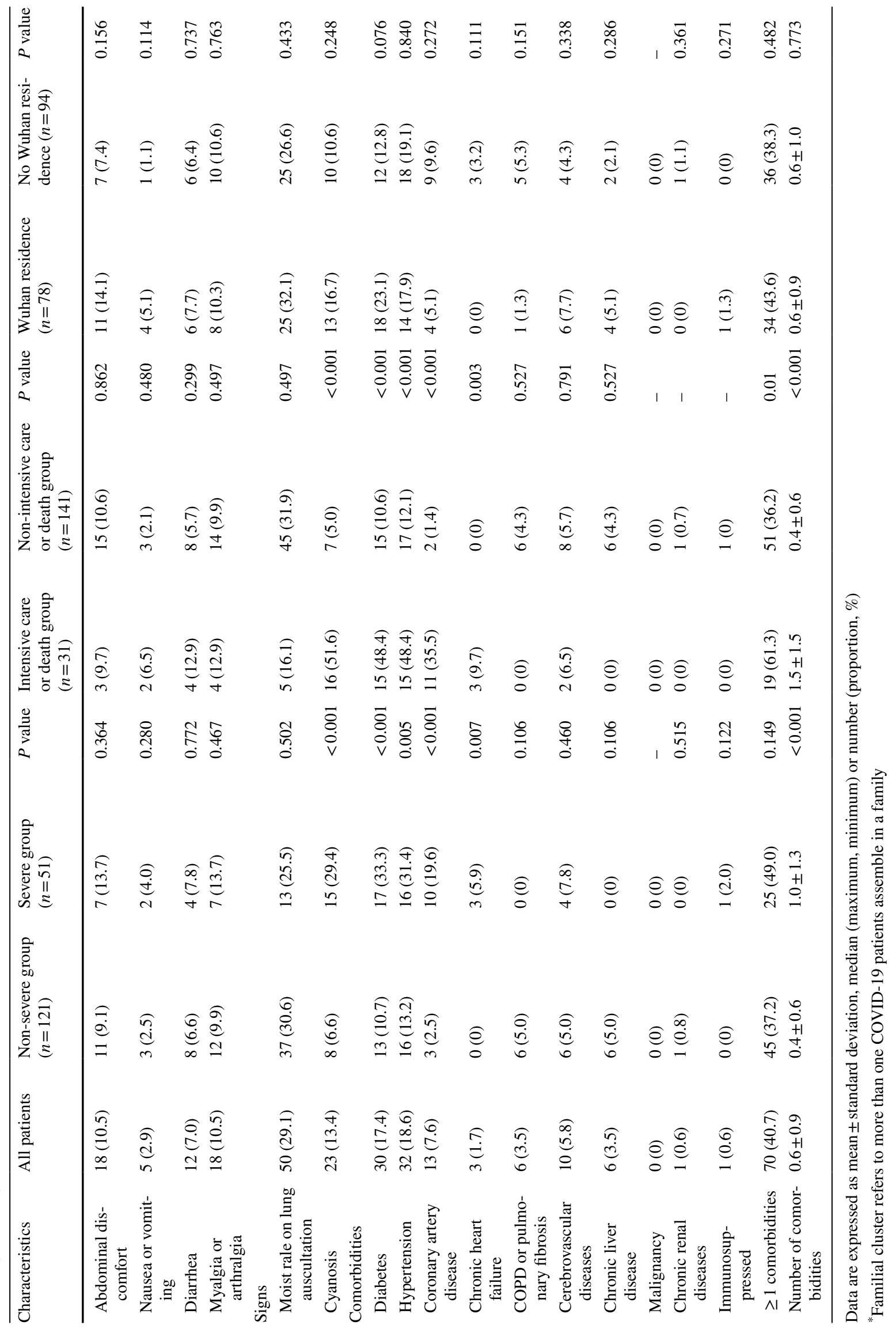




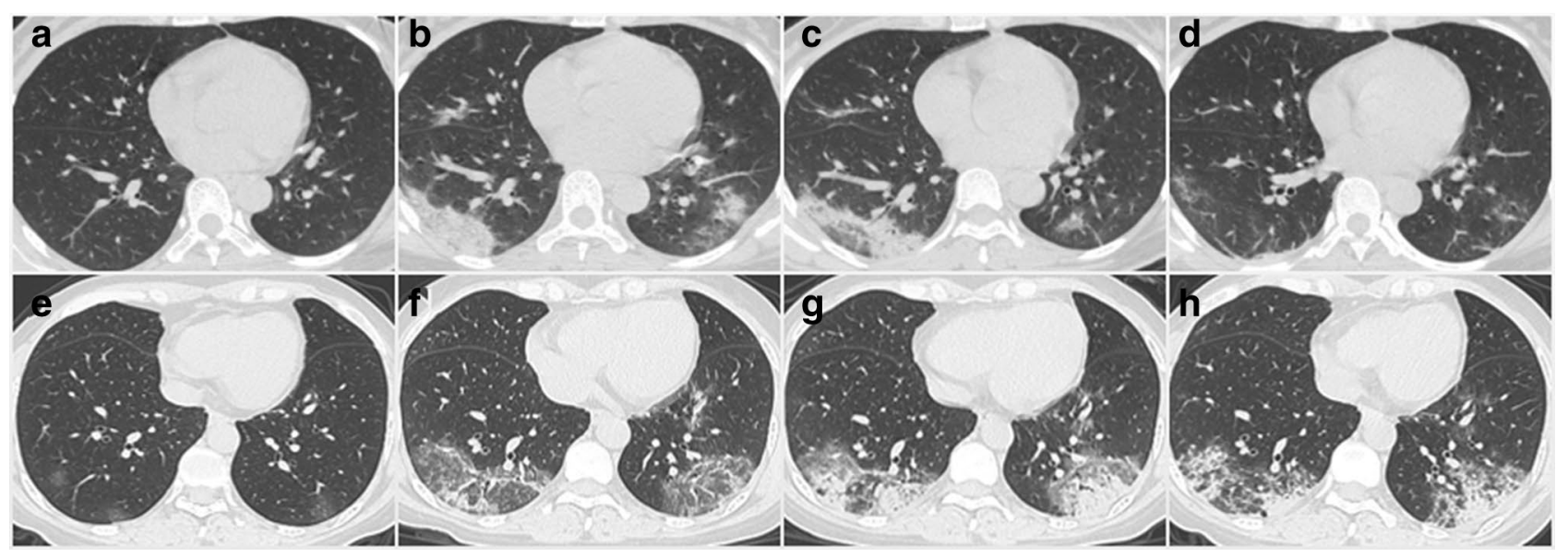

Fig. 1 Representative CT images of two cases with COVID-19. A-D: 31-year-old female. E-H: 28-year-old male

(from antiviral treatment) to virus clearance were $16.3 \pm 5.1$, $2.9 \pm 3.2,0.5 \pm 1.1,6.6 \pm 6.1,13.1 \pm 5.0$ days, respectively. $51.2 \%$ of all patients were admitted to the ICU, while $8.7 \%$ of all patients were subjected to IMV, ECMO or death. Complications include liver injury (11.6\%), myocardial injury $(8.7 \%)$, acute renal injury $(5.8 \%)$, the shock of any causes $(3.5 \%)$, pneumothorax $(1.7 \%)$ and major bleeding $(1.7 \%)$ that inquires immediate therapy and/or transfusion. $93.0 \%$ of all cases were discharged that included $86.3 \%$ severe cases, and $4.7 \%$ were still on treatment. The fatality rate was $2.3 \%$. The median CURB-65 score was 0 (range 0-2), while $79.1 \%$ of patients were 0 . The mean pneumonia severity index (PSI) score was $46.5 \pm 22.1$, and $87.2 \%$ of patients were classified with PSI grade of I or II (scoring $\leq 70$ ) score. The mean of MuLBSTA score was $7.0 \pm 4.3$, and $15.1 \%$ of all patients had a MuLBSTA score of $\geq 12$. At $72 \mathrm{~h}$ of admission, $23.3 \%$ of all patients underwent a clinical or radiological deterioration.

Statistical significance was observed in subgroup analysis between non-severe and severe group, and intensive care/ death group and non-intensive care/death group in antiviral regime, antiviral treatment switch, proportions of steroid use, maximum dose and duration of steroid use, antifungal use, IVIG, oxygen therapy, HFNC, NIV, IMV, ECMO and CRRT, overall co-infection and bacterial co-infection, the time to afebrile, CURB-65 score, PSI score and MuLBSTA score, complications, deterioration at $72 \mathrm{~h}$ and overall outcomes. However, the patients with or without Wuhan residence history do not show any differences except for their antiviral regimes.

\section{Risk factors of primary endpoint}

As shown in Fig. 2, Table 4 and Figure S3-S6, case type (severe or non-severe), proportions of lymphocyte and respiratory failure on admission are key risk factors of the primary endpoint. We did not perform subgroup analysis for death for a limited number of the death group.

\section{Discussion}

The current multi-center study depicts the overall characteristics and outcomes of 172 patients with COVID-19 in three cities of Henan province, where patients have free, fully access to all available medical resources. The median incubation period was 3.0 (95\% CI: 2.6-5.8, range: 1.0-17.0) days and is similar to those of previous cohorts [1-8] as well as a systemic review [18]. The average time from disease onset to the first visit and the time from disease onset to confirmation were $2.9 \pm 3.2$ and $0.5 \pm 1.1$ days, which are shorter than those in Wuhan [1-8]. These data demonstrate the availability of medical resources, which might be different from that in Wuhan in Feb 2020 but resembles more to the report of Zhejiang Province [7]. Another situation is that only $45.3 \%$ of all patients had a recent Wuhan exposure history (reside or travel in Wuhan), i.e. composed of third or fourth-generation cases. The third one is that by the time our cases were admitted much later than those of other studies of Wuhan when several editions of guidelines. Differences may thus be induced by the understandings and execution of triage and treatment guidelines by different doctors. Such discrepancies may partly explain the differences between this cohort and previous reports of Wuhan patients [1-8].

Household transmission has been documented in respiratory viruses such as influenza and SARS-CoV [12, 19-21]. According to several analytical researches, human coronaviruses such as MERS-CoV can survive on metal, glass or plastic surfaces for 9 days, but the virus were effectively inactivated by disinfectants, such as $62-71 \%$ ethanol, $0.5 \%$ hydrogen peroxide or $0.1 \%$ sodium hypochlorite [22]. Although our study did not provide direct evidences 


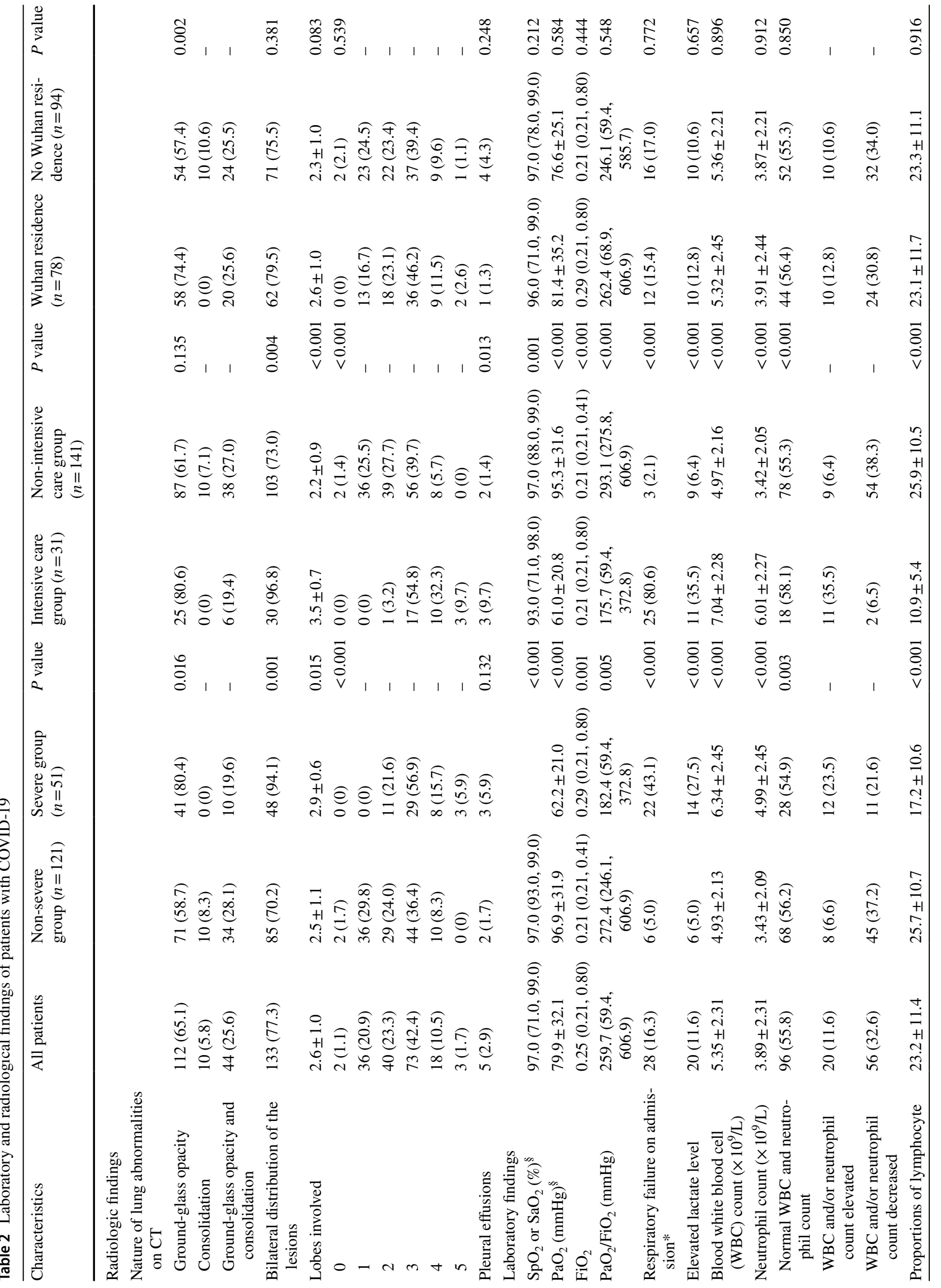




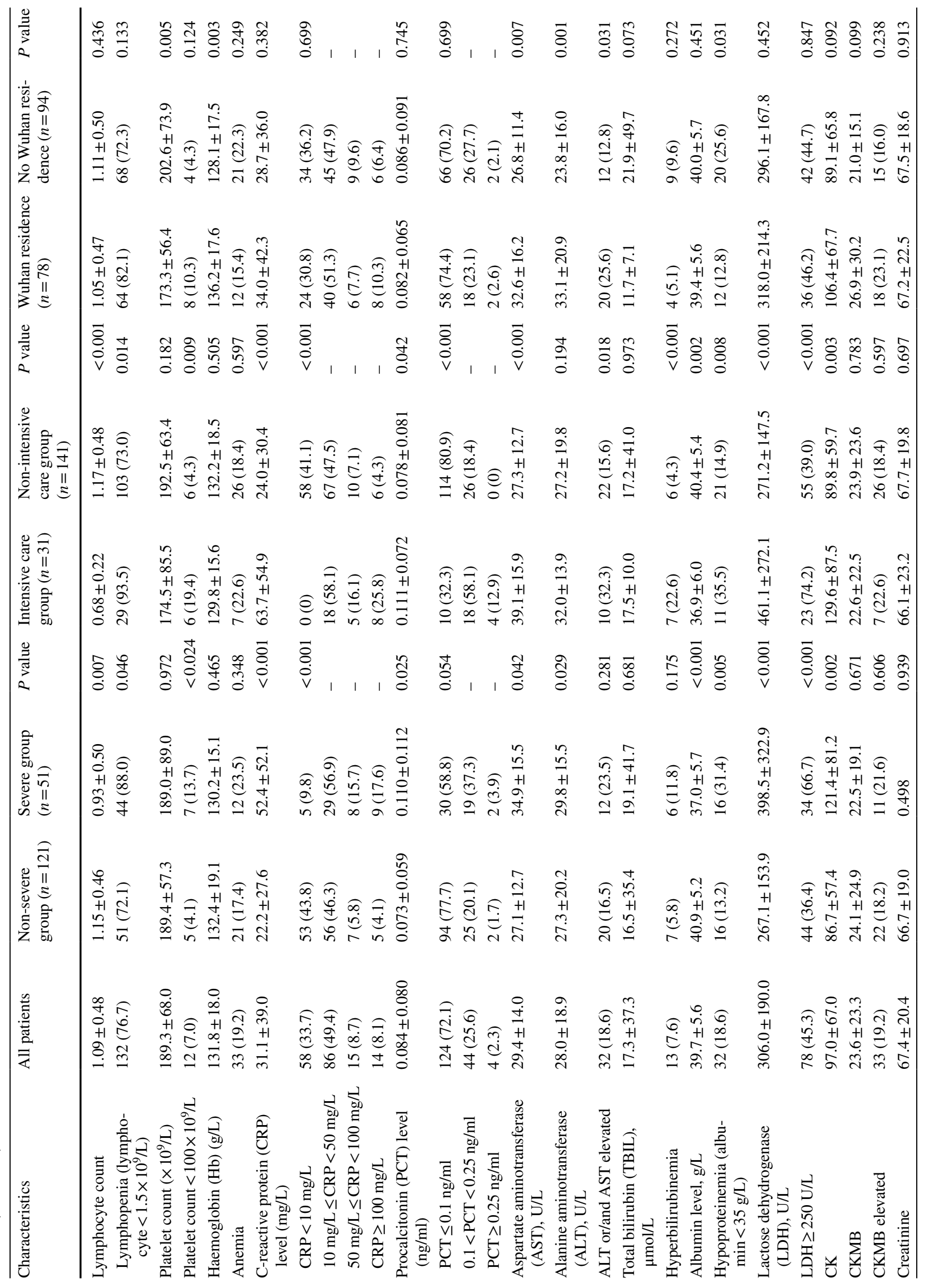




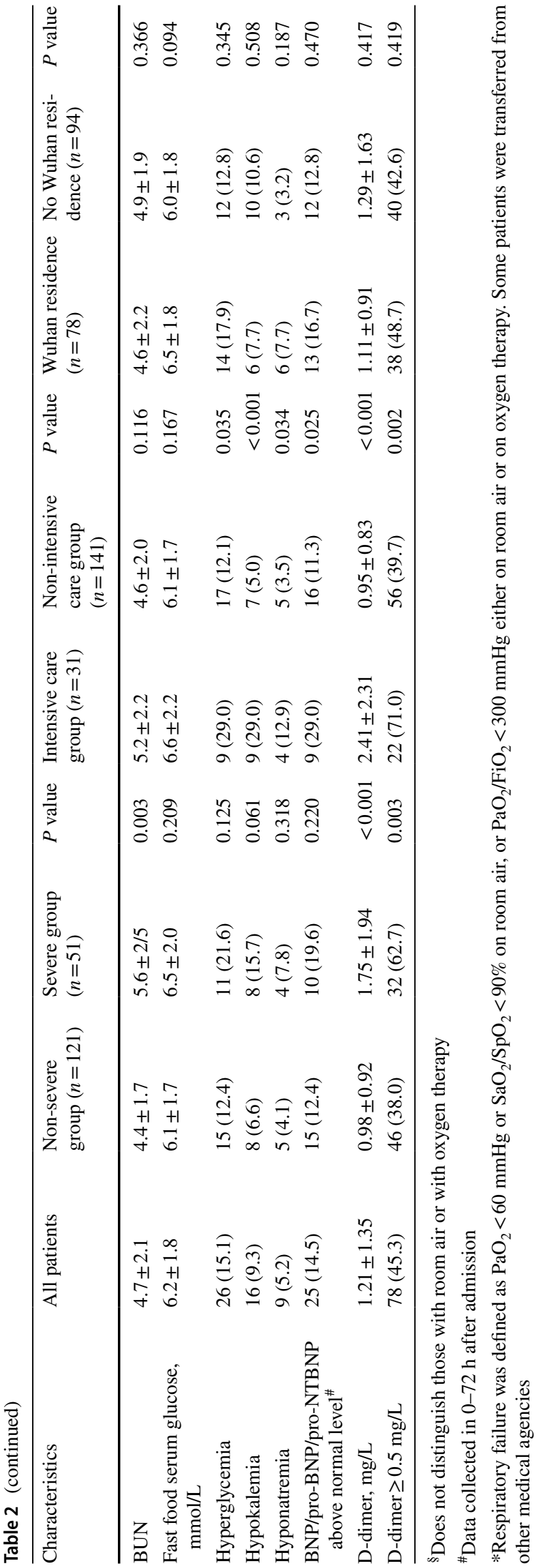

of household transmission, it's implicated by the fact that $26.7 \%$ of all patients had familial cluster, i.e. one relative or more to be diagnosed with SARS-CoV-2 infection. In a study about the family aggregation phenomenon, it was found that 5 persons in the same family were confirmed to be infected with COVID-19 after traveling to Wuhan, while another family which did not travel to Wuhan was infected with the virus several days after contacting with four family members [12]. In another study on family gatherings, COVID-19 carried by two family members might be transmitted to the children during a grand temple fair [13, 23]. In addition, some patients were asymptomatic while the viral loads are as likely to cause COVID-19 infection in asymptomatic patients as in symptomatic patients [13]. Interestingly, the proportion of the family cluster is similar in the Wuhan exposure group and non-Wuhan exposure group. Precise contact and familial cluster history were missing for all patients, so we could not give an exact picture of the household secondary attack rate in each patient. By estimation, the household secondary attack rate was $28.7 \%$. This rate seems to be much higher than that of SARS (reported to be $6.2 \%$ [20] and 10.2\% [21]) and H1N1 influenza (reported to be $10 \%$ [19]). Domestic isolation without identification of COVID-19 cases seems to be dangerous, given that COVID19 has $\mathrm{a} \sim 60 \%$ rate of occult asymptomatic infection and is more difficult to be identified. In this scenario, in terms of optimizing COVID-19 pandemic control by constraining household transmission, each patient shall be identified and isolated with medical care, and individual with an exposure history shall be isolated within a medical surveillance period away from their familial members. Furthermore, the R0 of SARS-CoV-2 is presumably 3.0 to 3.5 , but the average number of familial member infection is $2.2 \pm 1.6$ (95\% CI: 2.0-3.5, range: $0-4$ ). The gap might be attributed to both the reduction of virulence and transmissible capability and the strict measures undertaken by the local government and by the people, including traffic block and individual isolation, handwashing and wearing facial masks even at home.

According to this study, the gender distribution is similar in the current cohort as compared to most of other studies. In terms of gender ratio, there were 80 female patients in this study, accounting for $46.5 \%$ of the total proportion and $53.5 \%$ less than men. In previous studies on SARS and MERS, the proportion of female infection was low [24]. However, our analysis demonstrated that the proportion of male and female patients with COVID-19 was roughly the same without statistical difference. In another study of 138 patients diagnosed with COVID-19, $54.3 \%$ of the patients were male, which was similar to our statistics, with slightly more male patients than female patients [5].

The most common clinical features include fever, cough and lymphopenia that may have diagnostic or differential relevance. Wang et al. demonstrated that the most common 


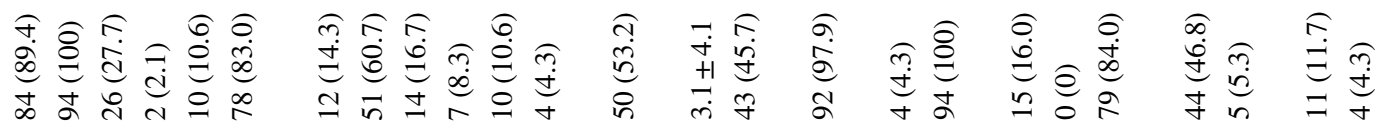

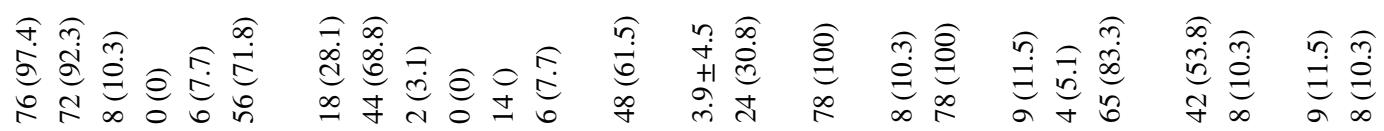

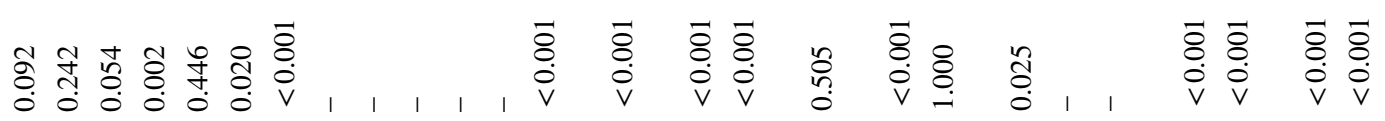

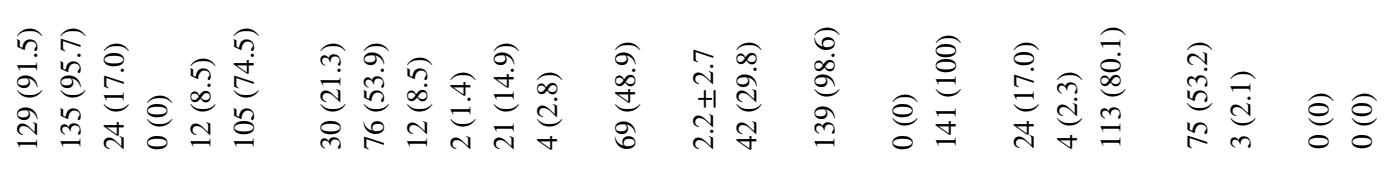

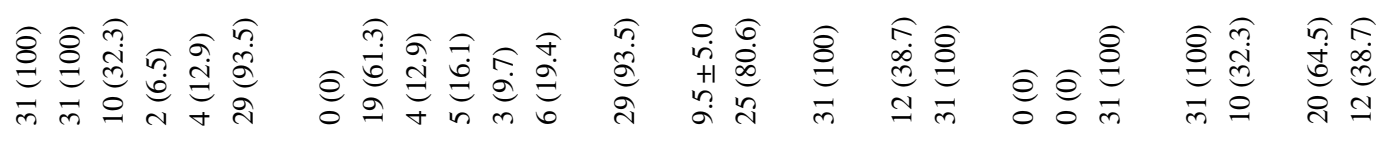

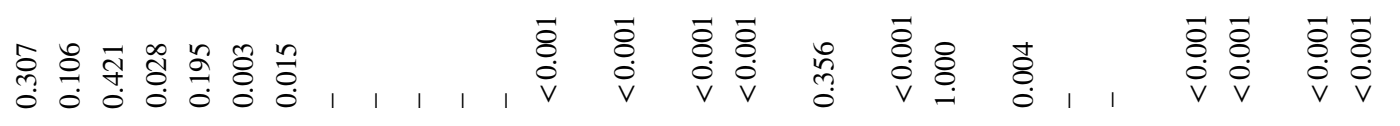

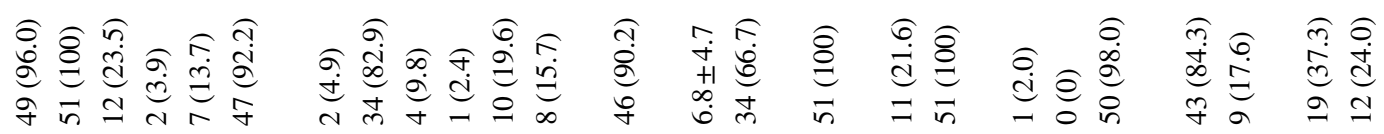

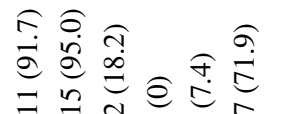

(ิ)

\section{(ิ)}

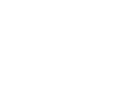

$$
\exists \vec{n}-0 \text { in } \rightarrow \text { a } 9 \text { I }
$$

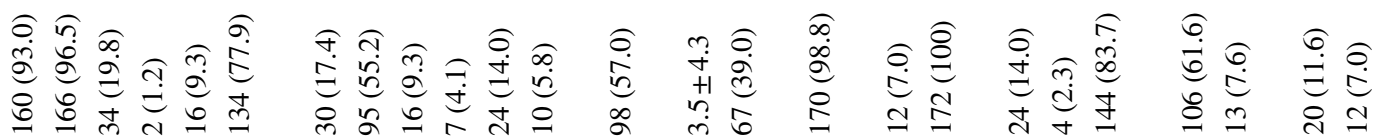




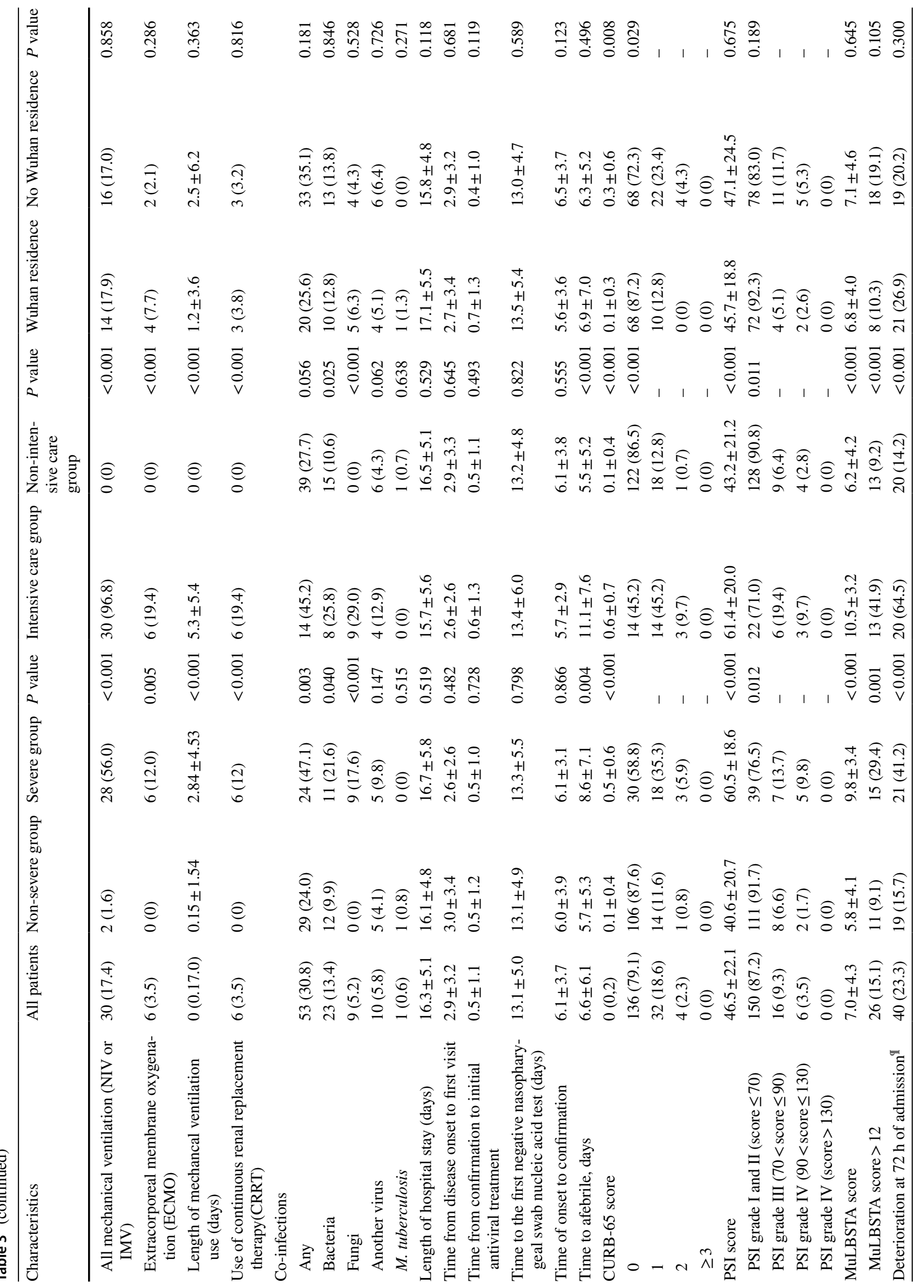




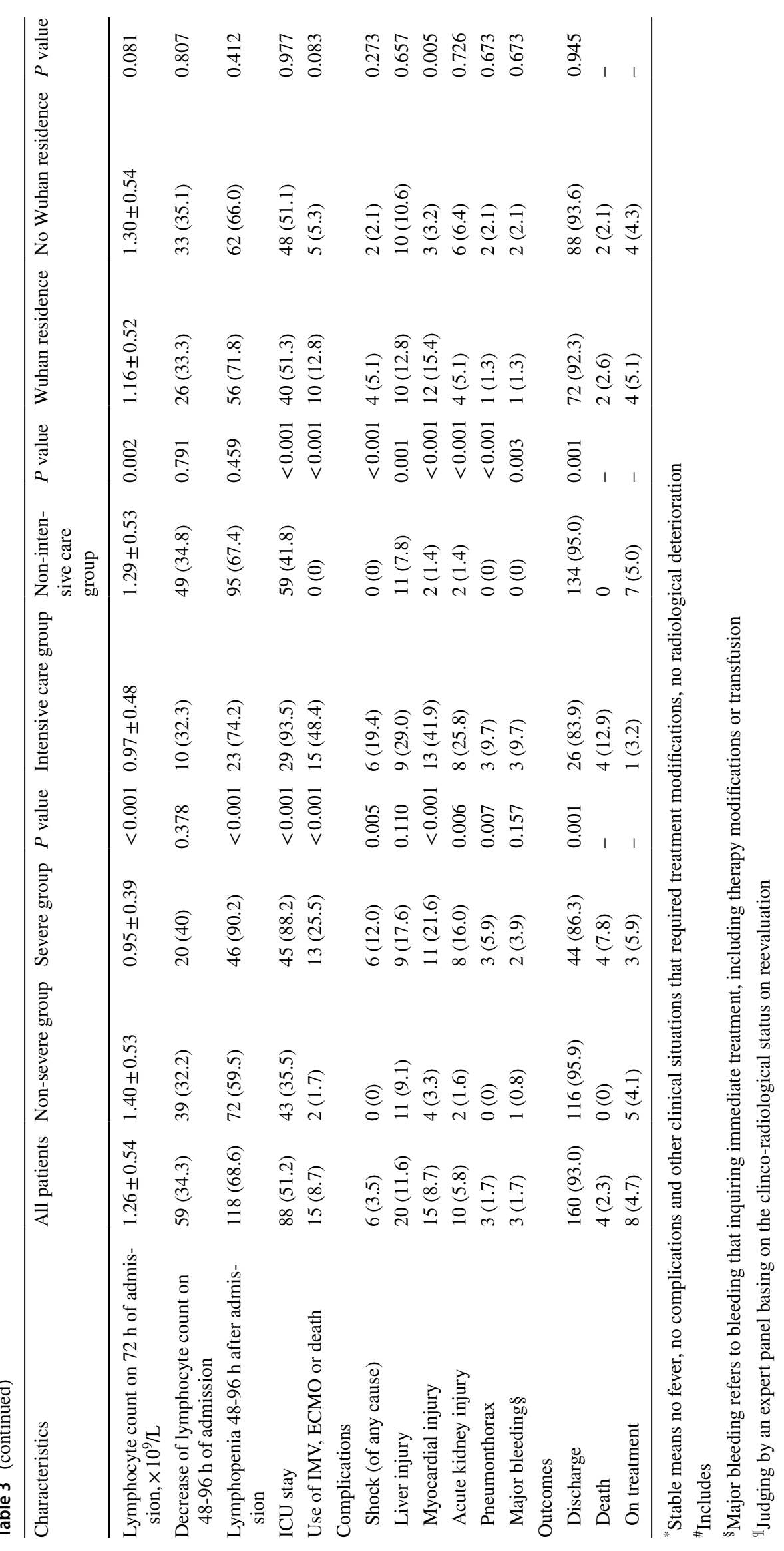


Fig. 2 Risk factors for invasive mechanical ventilation or ECMO or death as revealed by multivariate Logistic analysis included the case type (severe or nonsevere) (HR 1.126, 95\% CI 3.728-39.444), respiratory failure on admission (6.894, 95\% CI 1.364-35.748) and grade of lymphocyte proportion (HR 5.519, 95\% CI 1.70017.913)

Table 4 Univariate and multivariate regression analysis of risk factors for NIV/IMV/ $\mathrm{ECMO} /$ death

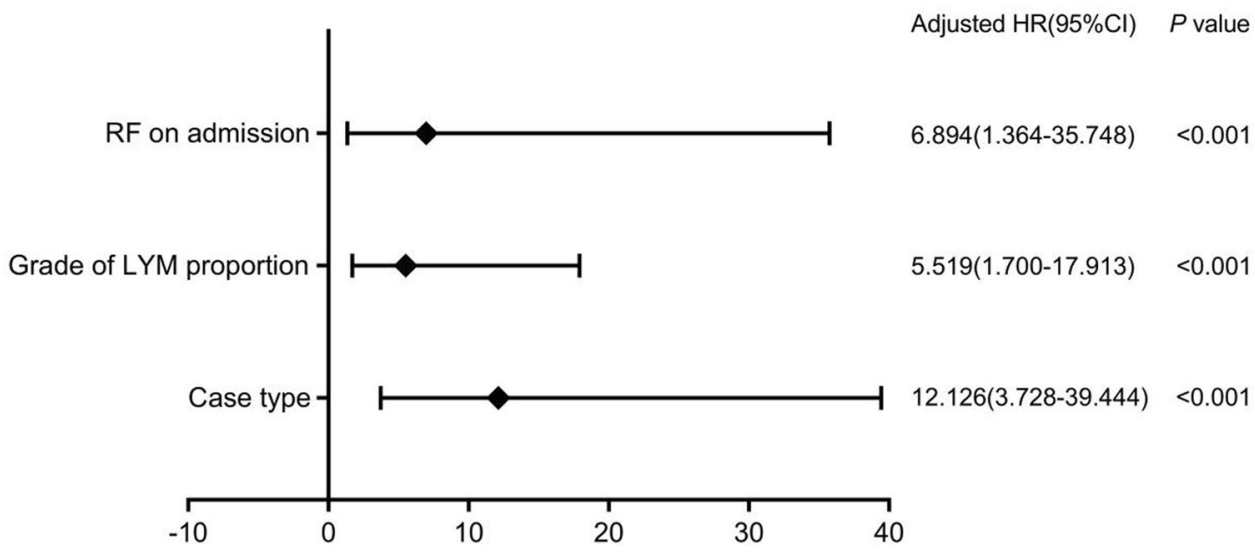

\begin{tabular}{|c|c|c|c|c|c|c|}
\hline & \multicolumn{3}{|c|}{ Univariate } & \multicolumn{3}{|c|}{ Multivariate } \\
\hline & OR & $95 \% \mathrm{CI}$ & $P$ value & OR & $95 \% \mathrm{CI}$ & $P$ value \\
\hline Case type & 24.128 & $8.442,68.960$ & $<0.001$ & 12.1 & $0.01,0.432$ & 0.005 \\
\hline Grade of LYM proportion & 6.018 & $3.291,11.004$ & $<0.001$ & 5.519 & $1.700,17.913$ & 0.004 \\
\hline RF on admission & 40.200 & $13.791,117.177$ & $<0.001$ & 6.984 & $1.364,35.748$ & 0.020 \\
\hline Number of lobes infiltrated & 0.15 & $0.072,0.316$ & $<0.001$ & 0.267 & $0.072,0.994$ & 0.267 \\
\hline
\end{tabular}

symptoms of 138 COVID-19 patients in Wuhan were fever (136 cases, 98.6\%), fatigue (96 cases, 69.6\%), dry cough (82 cases, 59.4\%), myalgia (48 cases, 34.8\%) and dyspnea (43 cases, $31.2 \%$ ) [5]. In addition, Chen et al. reported that the symptoms of 99 cases of COVID-19patients in Wuhan were characterized by fever ( 82 cases, $83 \%$ ), cough (81 cases, $82 \%$ ), shortness of breath (31 cases, $31 \%$ ), muscle soreness (11 cases, $11 \%$ ), unconscious ( 9 cases, $9 \%)$, headache ( 8 cases, $8 \%$ ), sore throat ( 5 cases, $5 \%$ ), runny nose (4 cases, $4 \%$ ), chest pain ( 2 cases, $2 \%$ ), diarrhea ( 2 cases, $2 \%$ ), nausea and vomiting (1 case, 1\%) [4].

Interestingly, abdominal symptoms, moist rale and cyanosis are uncommon. Bilateral involvement of GGO or GGO plus consolidation is most prevalent on CT images. $3.5 \%$ of all patients were CT-negative on admission, which differs from the same rate of Gan et al. [8]. The average number of lobes involved was $2.6 \pm 1.0 .40 .7 \%$ of the patients have at least one comorbid. In a study that evaluated the time period from symptom onset to first abnormal CT findings in patients with COVID-19, the results indicated that CT images were normal in $56 \%$ of patients within 2 days of symptom onset [25]. In addition, among patients with positive RT-PCR detection, the sensitivity of CT examination is high (86-97\%), while in patients with non-respiratory symptoms, the sensitivity is low (about 50\%) [26]. Furthermore, Bernheim er al. analyzed the CT results of COVID-19 patients, and found that $76 \%$ (25 cases) had bilateral lung lesions 3-5 days after the onset of symptoms, and 88\% (22 cases) had bilateral lung lesions 6-12 days after symptoms, indicating that the lesion scope gradually expanded with time [25]. Liver injury is the most prevalent complications, followed by myocardial injury, acute renal injury, shock, pneumothorax and major bleeding. These complications are further proved by laboratory test results of ALT, AST, CKMB, B type natriuretic peptide (Table 2). These data are consistent with previous studies and reviews [1-8, 12, 18-21, 27, 28]. These complications may develop before or after admission, but pneumothorax typically develops after NIV and IMV, and is supposed to be responsible for prolonged hospital stay. The serum levels of CRP and PCT are elevated in 66.3 and $27.9 \%$ in all cases, while $14.3 \%$ were complicated by bacteria co-infection. The rate of viral co-infection (notably influenza A or/and influenza A) is comparable to that of other studies [8]. M.bacterium was identified by fast-acid staining in 2 sputum samples of a 23-year old man with COVID-19, and this patient received standard antituberculosis therapy thereafter with isoniazid, rifampicin, pyrazinamide and ethambutol. He has had cough and expectoration more than 2 months before he had COVID-19, so we suppose a SARS-CoV-2 co-infection superimposed on tuberculosis infection in him. The issue of tuberculosis co-infection or relapse should be taken into account in developing countries with a high incidence of tuberculosis. An elevation of serum PCT, but not CRP, was associated with a bacterial infection. It's, therefore, concluded that procalcitonin is more specific than CRP in bacteria but not a viral infection. The events of major bleeding are rare and in parallel with prophylactic anticoagulative treatments or ECMO usage. On treatments and outcomes, $29.7 \%$ were severe cases, $16.3 \%$ had respiratory failure on 
admission, while $18.0 \%$ were subjected to IMV or ECMO, and the death rate is $2.3 \%$. Sanders et al. supported that the most promising treatment is Remdesivir [29]. Remdesivir is more effective against SARS-CoV-2 in vitro, but it has not been approved by the U.S. Food and Drug Administration. In addition, there is currently no evidence that oseltamivir is effective, and corticosteroid use and discontinuation of ACE2 inhibitors are not recommended. Another study also concluded that in patients with COVID-19, hydroxychloroquine reduced inflammatory responses and cytokine storms, but excessive use could lead to poisoning and death [30]. Neuraminidase inhibitors such as oseltamivir, peramivir and zanamivir are ineffective in the 2019-NCOV. Protease inhibitors such as lopinavir/ritonavir, combined with abidol, are effective in inhibiting early replication of SARS-CoV and have antiviral effects. The most universally used antiviral regime was lopinavir/ritonavir combined with aerosol IFN- $\alpha 1 b$, while darunavir and remdevisir were used in $10.5 \%$ of all cases. The antiviral regime was changed in 5.8\% of patients due to failure to initial treatment failure. Our data did not show statistical differences in different antiviral groups and severity groups in time to afebrile, time to virus clearance or length of stay. This retrospective data is in line with other reports on the effect of lopinavir/ritonavir on severe cases with COVID-19. Intravenous methylprednisolone was administered more in severe and intensive care cases as compared with non-severe and non-intensive care patients. However, corticosteroid use is not associated with unfavorable outcomes. Therefore, we would not give concrete data on the controversial use of glucocorticosteroids in COVID-19. IVIG and anti-inflammatory agents such as trazumab and baricitinib was supposed to be beneficial in some patients with COVID-19, but our data neither support nor oppose their usage.

Respiratory support is considered as an important supportive therapy. However, non-ICU patients are mainly treated with a nasal catheter or mask oxygen therapy, highflow oxygen inhalation and other methods, while ICU patients are treated with non-invasive mechanical ventilation, invasive mechanical ventilation and extracorporeal membrane oxygenation. Therefore, patients with severe disease and patients with severe events tend to occupy more medical resources [5]. In our research, the results indicated that oxygen therapy, high-flow nasal cannula oxygen therapy, NIV, IMV, ECMO was used in $61.6 \%, 7.6 \%$, $11.6 \%, 7.0 \%, 17.4 \%$ and $3.5 \%$ of all cases, respectively. Severe cases and the patients received intensive care tend to occupy more resources and cost more. However, our data showed that half of all patients had been ever admitted to the ICU, which include one-third of non-severe cases and is far higher than the rate of IMV and ECMO. We considered this as an unequal use or triage, if not, overuse, of medical resources. All the patients were admitted to the infectious disease department or ICU, but rather than to the respiratory department. Some patients were admitted to the ICU if the infectious department beds were occupied and unavailable, or if the patient was of older age (supposedly, $>80$ years), with more comorbidities or complications, or be transferred from another hospital. The average length of stay was significantly longer than that of time to afebrile but was closer to the time to viral clearance. An explanation is that nasal swab test for SARS-CoV-2 was not performed daily due to lack of test kits at that time, so a repeated nucleic acid test was performed after afebrile and/or CT resolution, making the time to viral clearance inaccurate. Shorter treatment duration seems to be feasible based on some recent studies.

Similar to previous studies, severe cases and patients subjected to intensive care were distinguished in clinical characteristics, prognosis and costs from non-severe cases and patients without intensive care, respectively. However, as for those with or without Wuhan exposure history, there were few discrepancies regarding history, symptoms, laboratory parameters and prognosis. To be inconsistent with other prognostic studies, CURB-65 score, PSI score or MuLBSTA score are not associated with composite unfavorable outcomes. These scoring systems were primarily developed for identifying patients with a high risk of death in CAP (CURB-65 and PSI score) or viral pneumonia (MuLBSTA score). The disease severity, the proportions of lymphocyte as well as respiratory failure on admission is possible risk factors for unfavorable outcomes. So, the triage of patients should keep in accord with the disease severity rather than with their exposure history or pneumonia severity scoring systems.

Our study has some limitations. The retrospective nature and relatively limited size of this study restrains its statistical confidence and interpretation of results, especially risk factors. Missing data exists, although not common, adding to research bias. The interpretation of the incubation period might be cautiously understood since the exposure history is somewhat secretive in $20 \%$ of all patients. Additionally, some patients are still being treated in hospital, and further follow-up is needed to give a more precise picture of their disease outcome or costs. Thirdly, we included five medical facilities that serve different people groups in 3 separate cities, 2 available with ECMO and 3 not. The immediate availability of medical resources may obscure the triage and treatment decision as well as disease outcome. Finally, the fees of all patients were covered by the government, which seems to be difficult to be replicated outside China.

In this retrospective analysis, clinical features and prognostic factors of 172 patients with COVID-19 were systematically analyzed, including age, gender, smoking history, exposure history in Wuhan, clinical symptoms, complications, radiological and laboratory indicators, therapeutic regimens, treatment time, risk factors for poor prognosis. 
The results showed that severe COVID-19, lymphocytopenia and respiratory failure were risk factors for COVID-19 progression.

Supplementary Information The online version contains supplementary material available at https://doi.org/10.1007/s13577-021-00499-y.

Funding Not applicable.

Data availability The data used to support the findings of this study are available from the corresponding author upon request.

\section{Compliance with ethical standards}

Conflict of interest The authors declare no conflict of interest to this work.

Ethical approval All procedures performed in studies involving human participants were in accordance with the ethical standards of the institutional committee and with the 1964 Helsinki declaration and its later amendments or comparable ethical standards. The research was approved by the academic committees of each hospital, Zhengzhou University and Nanyang Medical College.

Informed consent Informed consent was obtained from all individual participants included in the study.

\section{References}

1. Huang C, Wang Y, Li X, Ren L, Zhao J, Hu Y, et al. Clinical features of patients infected with 2019 novel coronavirus in Wuhan, China. Lancet. 2020;395(10223):497-506. https://doi. org/10.1016/s0140-6736(20)30183-5.

2. Li Q, Guan X, Wu P, Wang X, Zhou L, Tong Y, et al. Early transmission dynamics in Wuhan, China, of novel coronavirus-infected pneumonia. N Engl J Med. 2020;382(13):1199-207. https://doi. org/10.1056/NEJMoa2001316.

3. Zhu N, Zhang D, Wang W, Li X, Yang B, Song J, et al. A novel coronavirus from patients with pneumonia in China, 2019. N Engl J Med. 2020;382(8):727-33. https://doi.org/10.1056/NEJMo a2001017.

4. Chen N, Zhou M, Dong X, Qu J, Gong F, Han Y, et al. Epidemiological and clinical characteristics of 99 cases of 2019 novel coronavirus pneumonia in Wuhan, China: a descriptive study. Lancet. 2020;395(10223):507-13. https://doi.org/10.1016/s0140 $-6736(20) 30211-7$.

5. Wang D, Hu B, Hu C, Zhu F, Liu X, Zhang J, et al. Clinical characteristics of 138 hospitalized patients with 2019 novel coronavirus-infected pneumonia in Wuhan, China. JAMA. 2020;323(11):1061-9. https://doi.org/10.1001/jama.2020.1585.

6. [The epidemiological characteristics of an outbreak of 2019 novel coronavirus diseases (COVID-19) in China]. Zhonghua liu xing bing xue za zhi $=$ Zhonghua liuxingbingxue zazhi. 2020;41(2):145-51. https://doi.org/10.3760/cma.j.i ssn.0254-6450.2020.02.003.

7. Xu XW, Wu XX, Jiang XG, Xu KJ, Ying LJ, Ma CL, et al. Clinical findings in a group of patients infected with the 2019 novel coronavirus (SARS-Cov-2) outside of Wuhan, China: retrospective case series. BMJ. 2020;368:m606. https://doi.org/10.1136/ bmj.m606.
8. Guan WJ, Ni ZY, Hu Y, Liang WH, Ou CQ, He JX, et al. Clinical characteristics of coronavirus disease 2019 in China. N Engl J Med. 2020;382(18):1708-20. https://doi.org/10.1056/NEJMo a2002032.

9. Lu R, Zhao X, Li J, Niu P, Yang B, Wu H, et al. Genomic characterisation and epidemiology of 2019 novel coronavirus: implications for virus origins and receptor binding. Lancet. 2020;395(10224):565-74. https://doi.org/10.1016/s0140 $-6736(20) 30251-8$

10. Zhou P, Yang XL, Wang XG, Hu B, Zhang L, Zhang W, et al. A pneumonia outbreak associated with a new coronavirus of probable bat origin. Nature. 2020;579(7798):270-3. https://doi. org/10.1038/s41586-020-2012-7.

11. Lo Giudice R. The severe acute respiratory syndrome coronavirus-2 (SARS CoV-2) in dentistry. Management of biological risk in dental practice. Int J Environ Res Public Health. 2020. https://doi.org/10.3390/ijerph17093067.

12. Chan JF, Yuan S, Kok KH, To KK, Chu H, Yang J, et al. A familial cluster of pneumonia associated with the 2019 novel coronavirus indicating person-to-person transmission: a study of a family cluster. Lancet. 2020;395(10223):514-23. https:// doi.org/10.1016/s0140-6736(20)30154-9.

13. Zou L, Ruan F, Huang M, Liang L, Huang H, Hong Z, et al. SARS-CoV-2 viral load in upper respiratory specimens of infected patients. N Engl J Med. 2020;382(12):1177-9. https:// doi.org/10.1056/NEJMc2001737.

14. Yang X, Yu Y, Xu J, Shu H, Xia J, Liu H, et al. Clinical course and outcomes of critically ill patients with SARS-CoV-2 pneumonia in Wuhan, China: a single-centered, retrospective, observational study. Lancet Respir Med. 2020;8(5):475-81. https:// doi.org/10.1016/s2213-2600(20)30079-5.

15. Patel A, Jernigan DB. Initial public health response and interim clinical guidance for the 2019 novel coronavirus outbreak-United States, December 31, 2019-February 4, 2020. MMWR Morb Mortal Wkly Rep. 2020;69(5):140-6. https:// doi.org/10.15585/mmwr.mm6905e1.

16. Jin YH, Cai L, Cheng ZS, Cheng H, Deng T, Fan YP, et al. A rapid advice guideline for the diagnosis and treatment of 2019 novel coronavirus (2019-nCoV) infected pneumonia (standard version). Militar Med Res. 2020;7(1):4. https://doi.org/10.1186/ s40779-020-0233-6.

17. Younes N, Al-Sadeq DW, Al-Jighefee H, Younes S, Al-Jamal $\mathrm{O}$, Daas HI, et al. Challenges in laboratory diagnosis of the novel coronavirus SARS-CoV-2. Viruses. 2020. https://doi. org/10.3390/v12060582.

18. Lauer SA, Grantz KH, Bi Q, Jones FK, Zheng Q, Meredith $\mathrm{HR}$, et al. The incubation period of coronavirus disease 2019 (COVID-19) from publicly reported confirmed cases: estimation and application. Ann Intern Med. 2020;172(9):577-82. https:// doi.org/10.7326/m20-0504.

19. Cauchemez S, Donnelly CA, Reed C, Ghani AC, Fraser C, Kent CK, et al. Household transmission of 2009 pandemic influenza A (H1N1) virus in the United States. N Engl J Med. 2009;361(27):2619-27. https://doi.org/10.1056/NEJMoa0905 498.

20. Goh DL, Lee BW, Chia KS, Heng BH, Chen M, Ma S, et al. Secondary household transmission of SARS, Singapore. Emerg Infect Dis. 2004;10(2):232-4. https://doi.org/10.3201/eid1002.030676.

21. Wilson-Clark SD, Deeks SL, Gournis E, Hay K, Bondy S, Kennedy E, et al. Household transmission of SARS, 2003. CMAJ: Can Med Assoc J = Journal de l'Association Medicale Canadienne. 2006;175(10):1219-23. https://doi.org/10.1503/cmaj.050876.

22. Kampf G, Todt D, Pfaender S, Steinmann E. Persistence of coronaviruses on inanimate surfaces and their inactivation with biocidal agents. J Hosp Infect. 2020;104(3):246-51. https://doi. org/10.1016/j.jhin.2020.01.022. 
23. Chen T, Wu D, Chen H, Yan W, Yang D, Chen G, et al. Clinical characteristics of 113 deceased patients with coronavirus disease 2019: retrospective study. BMJ. 2020;368:m1091. https://doi. org/10.1136/bmj.m1091.

24. Wu F, Zhao S, Yu B, Chen YM, Wang W, Song ZG, et al. A new coronavirus associated with human respiratory disease in China. Nature. 2020;579(7798):265-9. https://doi.org/10.1038/s4158 6-020-2008-3.

25. Bernheim A, Mei X, Huang M, Yang Y, Fayad ZA, Zhang N, et al. Chest CT findings in coronavirus disease-19 (COVID-19): relationship to duration of infection. Radiology. 2020;295(3):200463. https://doi.org/10.1148/radiol.2020200463.

26. Kanne JP, Little BP, Chung JH, Elicker BM, Ketai LH. Essentials for radiologists on COVID-19: an update-radiology scientific expert panel. Radiology. 2020;296(2):E113-4. https://doi. org/10.1148/radiol.2020200527.

27. Bansal M. Cardiovascular disease and COVID-19. Diabetes Metab Syndr. 2020;14(3):247-50. https://doi.org/10.1016/j. dsx.2020.03.013.
28. Wong SH, Lui RN, Sung JJ. Covid-19 and the digestive system. J Gastroenterol Hepatol. 2020;35(5):744-8. https://doi.org/10.1111/ jgh. 15047.

29. Sanders JM, Monogue ML, Jodlowski TZ, Cutrell JB. Pharmacologic treatments for coronavirus disease 2019 (COVID-19): a review. JAMA. 2020;323(18):1824-36. https://doi.org/10.1001/ jama.2020.6019.

30. Sinha N, Balayla G. Hydroxychloroquine and COVID-19. Postgrad Med J. 2020;96(1139):550-5. https://doi.org/10.1136/postg radmedj-2020-137785.

Publisher's Note Springer Nature remains neutral with regard to jurisdictional claims in published maps and institutional affiliations. 